\title{
Teoría de la Formación por Etapas de las Acciones Mentales (TFEAM) en la Enseñanza y Aprendizaje del Concepto de Valencia Química
}

\author{
Aníbal Mendoza ${ }^{(1)}$, Diofanor Acevedo(2) y Candelaria N. Tejada ${ }^{(3) \star}$ \\ (1) Universidad del Norte, Departamento de Física, Km 5, Vía a Puerto Colombia. Barranquilla, \\ Atlántico-Colombia. \\ (2) Universidad de Cartagena, Facultad de Ingeniería, Programa de Ingeniería de Alimentos, \\ Avenida el Consulado, Calle 30 No. 48-152. Cartagena, Bolívar-Colombia. \\ (3) Universidad de Cartagena, Facultad de Ingeniería, Programa de Ingeniería Química, Avenida el \\ Consulado, Calle 30 No. 48-152. Cartagena, Bolívar-Colombia (e-mail: ctejadat@unicartagena.edu.co).
}

* Autor a quien debe ser dirigida la correspondencia

Recibido May. 6, 2015; Aceptado Jun. 17, 2015; Versión final Jul. 13, 2015, Publicado Feb. 2016

\begin{abstract}
Resumen
El objetivo de este trabajo fue aplicar la Teoría de la Formación por Etapas de las Acciones Mentales en la enseñanza y aprendizaje del concepto de valencia en química, evaluando su efecto en la apropiación del concepto. Se consideró el tiempo como un indicador de internalización del concepto de valencia y éste se relacionó con la cantidad de aciertos que realizó cada estudiante. Esto se hizo en una prueba de nueve ejercicios organizados en cinco categorías, con la finalidad de clasificar los resultados obtenidos en la prueba, tanto en el lenguaje para sí como en la etapa mental propiamente dicha. El análisis fue realizado mediante la aplicación del coeficiente de correlación lineal de Pearson. Este indica que un mayor número de estudiantes estuvieron más de 15 minutos en la etapa mental, mientras que en la etapa del lenguaje para sí la mayoría de los estudiantes estuvo sobre los 25 minutos.
\end{abstract}

Palabras clave: TFEAM, enseñanza, aprendizaje, didáctica, teoría de la actividad

\section{Theory of Stepwise Forming of Mental Actions (SFMA) in teaching and learning the concept of chemical valence}

\begin{abstract}
The objective of this project was to apply Theory of Stepwise Forming of Mental Actions in the teachinglearning process of the concept of chemical valence, evaluating its effect in the appropriation of the concept. Time was considered as an indicator of the valence concept's internalization and this was related to the quantity of successes that each student achieved. This was done in a test of nine exercises, organized in five categories with the aim of classifying the results in both the own language as well as in the mental stage. The analysis was carried out by the application of the Pearson's lineal correlation coefficient. This showed that more students were over the 15 minutes in the mental stage; while in the stage of own language the majority of the students spent more than 25 minutes.
\end{abstract}

Keywords: SFMA, teaching, learning, didactics, activity theory 


\section{INTRODUCCIÓN}

Respecto al concepto de Valencia Química, se identifican dos aspectos que los autores consideramos como puntos de inflexión en su enseñanza y aprendizaje, identificando con ello un problema que merece atención, que involucra aspectos tanto de orden pedagógico como didáctico relacionado con la forma como lo aprenden los estudiantes, dado lo abstracto del mismo, que por lo general conlleva a ignorar su trascendencia, y su relación con otros conceptos imprescindibles para la comprensión de la química. Es así como la enseñanza del concepto de valencia química, exige que las nuevas ideas deben estar relacionadas subordinadamente con conocimientos previos tales como: concepto de átomo, estructura electrónica del átomo, la regla de Hund, el principio de exclusión de Paulin, conceptos de anión y catión, y generalidades de tabla periódica (Tejada et al., 2013b). De otra parte está la manera como lo enseñan la mayoría de los docentes; que sin duda influye significativamente en que los estudiantes hagan una apropiación genuina del concepto, como se muestra en el estudio realizado por Tejada et al., (2013a), quienes estudiaron la forma como los estudiantes expresan los modelos de átomos, quedando en evidencia la influencia de las diferentes formas como enseñan los docentes y las diversas formas y estilos como aprende los estudiantes. En este contexto la necesidad de didácticas que favorezcan la enseñanza aprendizaje del concepto de valencia se presenta aplicar la Teoría de la Formación por Etapas de las Acciones Metales (TFEAM) como una alternativa donde los conceptos se enseñan de manera deductiva a través de las siguientes etapas correspondientes a las formas de la acción. Primero se explica la etapa orientadora de la acción y se enuncian las características esenciales y no esenciales del concepto, que se va enseñar. Seguidamente se presentan situaciones en forma material o materializada para enseñar las características del concepto. De igual forma se presentan situaciones para que los estudiantes describan las características del concepto en estudio en forma verbal externa.

Según Talízina (1988) en la formación por etapas de las acciones mentales hay que indicar dos circunstancias. En primer lugar, en dependencia del tipo de acción se somete a la elaboración por etapas toda la acción o sólo su parte orientadora. Esto último tiene lugar durante la formación, ante todo, de distintas habilidades deportivas y de producción, del saber de escribir. En segundo lugar, la realización de la acción hasta la etapa mental no significa el fin de su desarrollo. Las etapas analizadas son el camino de lo externo a lo interno; pero la formación de las acciones mentales y de los conceptos no constituye un objetivo en sí; la TFEAM se fundamenta en la teoría de la actividad de Vigostky analizada por Galperin (1995) y Talízina (1988) y explica el paso de la actividad externa a la actividad interna en la mente del hombre y la aplicó de manera novedosa en el proceso de aprendizaje. Esta teoría plantea que para lograr lo anterior, el sujeto debe pasar por determinados momentos de la actividad, conformada por la orientación y la ejecución en el proceso de asimilación.

Según Galperin (1995) las diferentes etapas de los momentos funcionales de la actividad son: En una primera etapa (etapa de elaboración del esquema de la base orientadora de la acción) los estudiantes reciben las explicaciones necesarias sobre el objetivo de la acción, su objeto, el sistema de puntos de referencia. Es la etapa de conocimiento previo de la acción y de las condiciones de su cumplimiento: la etapa de la elaboración del esquema de la base orientadora de la acción, en ella, el alumno obtiene conocimientos sobre el objeto de estudio y sobre la actividad y el orden en que se va a realizar, de las acciones y las operaciones que la componen. En una segunda etapa (etapa de la formación de la acción en forma material o materializada), los estudiantes ya cumplen la acción, pero por ahora en forma material (o materializada) externa con el despliegue de todas las operaciones que forman parte de ella. En una forma así, se realizan las partes orientadora, ejecutora y de control de la acción. Esta etapa permite a los alumnos asimilar el contenido de la acción, y al profesor, realizar un control objetivo del cumplimiento de cada una de las operaciones que forman parte de la acción. En esta etapa el alumno resuelve las tareas realizando la actividad en forma externa, con el apoyo de la forma material o materializada del objeto y con el acompañamiento del docente. Luego sigue una tercera etapa (etapa de la formación de la acción como verbal externa), en la que los elementos de la acción se presentan en la forma verbal externa, la acción pasa por la generalización, pero aún sigue siendo no automatizada ni reducida. En esta etapa el habla comienza a cumplir una nueva función. En la primera y segunda etapa según Galperin (1995)el habla servía principalmente de sistema de indicaciones que se descubrían directamente en la percepción; la tarea del alumno consistía no en comprender las palabras, sino comprender y dominar los fenómenos. Mientras que ahora el habla se convierte en portadora independiente de todo el proceso: tanto de la tarea como de la acción. Aquí los elementos de la actividad, deben presentarse en forma oral o escrita. La cuarta etapa (etapa de la formación de la acción en el lenguaje externo "para sí") se distingue de la anterior en que la acción se realiza en silencio, sin escribirla: como interpretación para sus adentros. Al principio, la acción según las restantes características (el carácter desplegado, de conciencia, de generalización) no difiere de la etapa anterior. Sin embargo, al adquirir la forma mental, la acción empieza a reducirse y automatizarse muy rápidamente, adquiriendo la forma de la acción según la establece. 
Finalmente una quinta etapa (etapa de la formación de la acción en el lenguaje interno) corresponde a la realización de la acción en el plano mental, al principio se realiza en forma lenta, pero después la actividad se automatiza y se realiza en forma rápida para convertirse en una actividad de pensamiento. En esta etapa se le deben dar al estudiante tareas de mayor complejidad que exijan soluciones creativas. La acción adquiere muy rápidamente un desarrollo automático, se hace inaccesible al auto observación. Ahora se trata del acto del pensamiento, donde el proceso está oculto, y se abre a la conciencia sólo el producto de este proceso; "en la acción mental formada escribe Galperín (1995), casi todo su contenido real abandona la conciencia, y lo que queda en ella no puede ser comprendido correctamente sin la relación con los demás"; Galperín (1995) indica la necesidad de introducir una etapa más, donde la tarea principal consiste en la creación de una motivación necesaria en el alumno. Independientemente de si la solución de la tarea dada constituye o no una etapa independiente, debe estar asegurada la existencia de motivos necesarios para que los alumnos adopten la tarea de estudio y cumplan la actividad que le es adecuada. Si esto no ocurre es imposible la formación de las acciones y de los conocimientos que entran en ellas (en la práctica de la enseñanza es bien sabido que si el alumno no quiere estudiar, es imposible enseñarle). Para crear una motivación positiva se utiliza comúnmente la creación de situaciones problemáticas, que es posible solucionar por medio de la acción cuya formación ha de emprenderse.

Coherente con lo anterior vale la pena resaltar algunos trabajos actuales que reportan la utilidad de la aplicación de la teoría de la formación por etapas de las acciones mentales; como el reportado por Tejada et al., (2013b) quienes abordaron el tema de la enseñanza de la química basada en la formación por etapas de acciones mentales; el fundamento teórico para el desarrollo de la didáctica propuesta, se encuentra en la concepción dialéctica del conocimiento y las ideas de la teoría de la actividad y la enseñanza, basada en la formación por etapas de las acciones mentales, bajo el esquema del aprendizaje subordinado. Soportada en las teorías de Vigotsky, Leantiev, Galperin, Talizina y Ausubel. Finalmente, se propone un método para que el estudiante realice la reconciliación integradora, que le permitirá desarrollar una mayor capacidad para la asimilación significativa de conceptos relacionados con la química, de manera específica el concepto de valencia. También Gnitetskaya (2014), quien Estudio los fundamentos psicológicos para la disposición de un módulo de física, Aplicando la teoría de la formación por etapas de las acciones mentales (TFEAM), la estructura del módulo desarrollado contiene elementos necesarios para la ejecución de los pasos de la teoría TFEAM, en el proceso de la enseñanza de la física y se demostró que la enseñanza de la física sugerida no contradice la eficiencia de los factores psicológicos del proceso de enseñanza. De una forma parecida y en la misma área del conocimiento; Rodríguez-Llerena y Llovera-González (2014), quienes realizaron un estudio comparativo de las potencialidades didácticas de las simulaciones virtuales y de los experimentos reales en la enseñanza de la Física General para estudiantes universitarios de ciencias técnicas, donde cobra fuerza la didáctica desde una perspectiva investigativa y que se centra en la pregunta guía. Presentando resultados de una investigación realizada en dos cursos académicos con el fin de fundamentar psicodidacticamente en qué medida las simulaciones virtuales de experimentos docentes, pueden contribuir objetivamente a aumentar la calidad en el aprendizaje de la física general universitaria en comparación con la realización de los experimentos docentes reales. Considerando que las simulaciones virtuales y de los experimentos reales, son la forma materializada que favorece la enseñanza aprendizaje de conceptos.

\section{MATERIALES Y MÉTODOS}

En el estudio participaron 40 estudiantes de segundo semestre de ingeniera química de la asignatura de química inorgánica, de una universidad de la ciudad de Cartagena de Indias, con edades que oscilan entre 16 y 19 años; estos estudiantes son bachilleres de colegios públicos y privados, oriundos de diferentes ciudades de la Costa Caribe, de diferentes estratos. Además de, tres investigadores, quienes diseñaron en equipo los instrumentos que se aplicaron en la implementación de la didáctica basada en la TFEAM.

Se partió de la internalización del concepto de valencia química, en forma material (paso de lo concreto a lo abstracto), lo cual fue complejo, dado lo abstracto del concepto de valencia en química; para lo cual los estudiantes sustentaron mediante la técnica de la puesta en común, modelos de moléculas, en donde de manera consciente, no memorística explicaron el concepto completo de valencia química, es decir que incluía valencia primaria y valencia secundaria. En esta misma etapa se combinó la etapa verbal externa, tanto oral como escrita. Seguidamente se presentaron situaciones para que los estudiantes puedan establecer la presencia o ausencia de las características del concepto; esto lo debe establecer el estudiante en forma individual, lo cual, constituye la forma de la acción de "lenguaje para sí", con la ayuda sólo de una tarjeta que contiene en forma desplegada las características del concepto, ley o principio. También se debe presentar otra situación problemática en la que los estudiantes deben resolverla en forma individual, pero a diferencia de la anterior, sin ayuda de la tarjeta que contiene las características. Esta etapa es propiamente la forma mental de la acción. Por último está la etapa de motivación, debe estar presente durante todo el proceso, por ello le hemos denominado etapa transversal. Es de notar que en cada una de estas etapas se 
debe ejercer un control, lo cual constituye la forma de evaluación por proceso. Es importante recordar aquí, la utilidad que nos brindaría las técnicas de Assessment aplicadas en el salón de clase (Angelo y Cross, 1993).

En una etapa previa durante el desarrollo del proceso de enseñanza aprendizaje del concepto de valencia el docente desarrolló la basé orientadora de la acción la cual consistió principalmente en llevar paulatinamente a los estudiantes a la identificación de las características del concepto de valencia, de tal forma que pudieran diferenciar claramente los conceptos de valencia primaria y valencia secundaria; así como la explicación de la existencia de moléculas inorgánicas sencillas y complejas con centros metálicos, apoyándonos en la comprensión de las herramientas fundamentales halladas en la tabla periódica, ubicando los elementos representativos y entender la manera de formar enlaces; comprendiendo de esta forma la valencia primaria; así mismo la ubicación e identificación de elementos de transición y transición interna y la manera como se forman las moléculas con centros metálicos y diversos tipos de ligandos; buscando que comprendieran de esta manera la valencia secundaria, persiguiendo con esto que los estudiantes construyeran un concepto de valencia química de manera integrada y a la vez amplia. Las características del concepto fue abordado por el docente con la ayuda del mentefacto del concepto de valencia y el mapa conceptual. La prueba consistió en un test con 9 ejercicios relacionados con la identificación y formulación de diferentes tipos de moléculas inorgánicas sencillas y complejas, también se incluyeron algunos relacionados con el tipo de enlace químico, numero de oxidación y numero de coordinación; lo anterior se complementa con lo descrito en la tabla 1.

Tabla 1: Descripción de las etapas abordadas en el modelo de la TFEAM

\begin{tabular}{|c|c|}
\hline Etapas de la TFEAM & Síntesis del desarrollo de cada etapa del modelo \\
\hline la base orientadora de la acción & Impartida por el docente apoyado en mentefacto y mapas conceptuales \\
\hline $\begin{array}{c}\text { la formación de la acción en forma } \\
\text { material o materializada }\end{array}$ & Moléculas elaboradas con esferas de icopor y palillos \\
\hline $\begin{array}{c}\text { la formación de la acción como } \\
\text { verbal externa }\end{array}$ & $\begin{array}{c}\text { Los estudiantes explicaron el concepto completo de valencia química } \\
\text { apoyados en los diferentes tipos de moléculas elaboradas en icopor, tanto } \\
\text { verbal como escrita. }\end{array}$ \\
\hline $\begin{array}{c}\text { de la formación de la acción en el } \\
\text { lenguaje externo "para sí }\end{array}$ & $\begin{array}{c}\text { El estudiante asimila en silencio y mentalmente las etapas anteriores para lo } \\
\text { cual se le da un tiempo determinado. }\end{array}$ \\
\hline $\begin{array}{c}\text { la formación de la acción en el } \\
\text { lenguaje interno }\end{array}$ & $\begin{array}{c}\text { El estudiante se enfrenta a una tarea de mayor complejidad identificando y } \\
\text { formulando moléculas diferentes a las creadas en la forma material e } \\
\text { identificando las características del concepto. }\end{array}$ \\
\hline
\end{tabular}

\section{RESULTADOS Y DISCUSIÓN}

Los resultados de la etapa mental y del lenguaje para sí se presentan en las Tablas 2, 3, 4 y 5. Inicialmente se les entregó a los estudiantes el test acompañado con la ficha de características del concepto de valencia; la prueba dio inició a las 12:10 PM y a partir de esta hora se tomó el tiempo que demoraba un estudiante resolviendo el test.

Tabla 2: Relación entre número de estudiantes y tiempo de entrega del test acompañado con ficha de características

\begin{tabular}{|c|c|c|}
\hline $\begin{array}{c}\text { Hora de entrega del test resuelto por el } \\
\text { estudiante (PM) }\end{array}$ & $\begin{array}{c}\text { Tiempo que demora el } \\
\text { estudiante para resolver el test (minutos) }\end{array}$ & Cantidad de estudiantes \\
\hline $12: 25$ & 15 & 3 \\
\hline $12: 30$ & 20 & 26 \\
\hline $12: 35$ & 25 & 5 \\
\hline $12: 40$ & 30 & 40 \\
\hline
\end{tabular}

Con la finalidad de agotar la etapa mental, seguidamente se realizó una prueba utilizando el mismo modelo pero con la diferencia que no se les entregó la ficha con las características. La actividad se inició a las 12:45 PM; los tiempos obtenidos se pueden observar en la Tabla 3 . En las Tablas 2 y 3 se observa que los estudiantes cuando aplican las propiedades del concepto de valencia en la etapa mental, la gran mayoría tardan menos tiempo para resolver la prueba, que cuando la resuelven en la etapa del lenguaje, lo cual era de esperarse según la teoría de la formación por etapas de las acciones mentales, en la que se sustenta nuestra didáctica, tal como se concibe en el carácter asimilado de la acción en donde se plantea que el cumplimiento de la acción se realiza haciendo conciencia de cada una de las operaciones, de modo lento; 
pero paulatinamente la acción se va automatizando y el ritmo de su cumplimiento aumenta (Talízina, 1988). En este sentido notamos, que un mayor número de estudiantes estuvieron sobre los 15 minutos en la etapa mental, mientras que en la etapa del lenguaje para sí, el mayor número de estudiantes estuvo sobre los 25 minutos. Lo anterior puede ser evidencia de que probablemente hubo internalización por parte de los estudiantes de las propiedades del concepto de valencia.

Tabla 3: Relación entre número de estudiantes y tiempo de entrega de la prueba con ficha sin características

\begin{tabular}{|c|c|c|}
\hline $\begin{array}{c}\text { Hora de entrega del test resuelto por el } \\
\text { estudiante (PM) }\end{array}$ & $\begin{array}{c}\text { Tiempo que demora el estudiante para resolver } \\
\text { el test (minutos) }\end{array}$ & $\begin{array}{l}\text { Cantidad de } \\
\text { estudiantes }\end{array}$ \\
\hline $12: 45$ & 0 & 1 \\
\hline $12: 55$ & 10 & 1 \\
\hline $1: 00$ & 15 & 37 \\
\hline $1: 15$ & 30 & 1 \\
\hline \multicolumn{2}{|r|}{ Total } & 40 \\
\hline
\end{tabular}

Además de considerar el tiempo como un indicador de internalización del concepto de valencia, también consideramos importante relacionar la cantidad de aciertos que realiza cada estudiante. Teniendo en cuenta que la prueba tenía 9 ejercicios, organizamos cinco categorías, con la finalidad de poder estratificar los resultados obtenidos en la prueba, tanto en el lenguaje para sí, como en la etapa mental propiamente dicha, quedando como se presenta en las Tablas 4 y 5.

Tabla 4: Relación entre número de estudiantes y número de aciertos de la prueba con ficha de características

\begin{tabular}{|c|c|c|}
\hline Categoría & Número de aciertos & Número de estudiantes \\
\hline 1 & $8-9$ & 4 \\
\hline 2 & $6-7$ & 20 \\
\hline 3 & $4-5$ & 7 \\
\hline 4 & $1-3$ & 7 \\
\hline 5 & 0 & 2 \\
\hline \multicolumn{2}{|c|}{} & 40 \\
\hline
\end{tabular}

Tabla 5: Relación entre número de estudiantes y número de aciertos de la prueba con ficha sin características

\begin{tabular}{|c|c|c|}
\hline Categoría & Número de aciertos & Número de estudiantes \\
\hline 1 & $8-9$ & 11 \\
\hline 2 & $6-7$ & 20 \\
\hline 3 & $4-5$ & 5 \\
\hline 4 & $1-3$ & 2 \\
\hline 5 & 0 & 2 \\
\hline \multicolumn{2}{|c}{} \\
\hline
\end{tabular}

También, dentro del análisis descriptivo, consideramos útil evaluar estadísticamente dos variables utilizadas para medir el grado de internalización del concepto de valencia, tales como el tiempo de resolución de la prueba (variable independiente $X$ ) y número de aciertos de la prueba por cada estudiante (variable dependiente Y). Dada la existencia de dos variables cuantitativas continuas, se aplicó el coeficiente de correlación lineal de Pearson ( $r$ ), fue calculado con la Ecuación (1), dónde $r$ es el coeficiente de correlación de Pearson; $\mathrm{N}$ es el número total de pares de puntajes $\mathrm{X}$ y $\mathrm{Y} ; \mathrm{X}$ es el puntaje crudo en la variable $\mathrm{X} ; \mathrm{Y}$ es el puntaje crudo en la variable $Y$. Para interpretar los resultados obtenidos se tuvo en cuenta que los coeficientes de correlación se encuentran generalmente entre $-1,00$ y +1,00(Borda, 2005).

$$
r=\frac{\mathrm{N} \sum \mathrm{XY}-\left(\sum \mathrm{X}\right)\left(\sum \mathrm{Y}\right)}{\sqrt{\left[\mathrm{N} \sum \mathrm{X}^{2}-\left(\sum \mathrm{X}\right)^{2}\right]\left[\mathrm{N} \sum \mathrm{Y}^{2}-\left(\sum \mathrm{Y}\right)^{2}\right]}}
$$

Para la prueba con ficha de características el ( $r$ ) tiene un valor absoluto de 0,26 con lo que interpretamos que existe una correlación débil a moderada entre el tiempo de ejecución de la prueba y el número de aciertos, es decir a menor tiempo gastado por el estudiante para resolver la prueba, mayor es el número de aciertos. Se observa que hay una correlación débil, lo que se puede interpretar como que, no hubo una internalización profunda del concepto, debido a que está relacionada directamente con el número de aciertos. Para la prueba sin ficha de características el valor absoluto del $(r)$ obtenido fue de 0,30 , aunque 
sigue comportándose como una correlación débil a moderada, es muy significativo para nuestro análisis por dos razones, la primera, porque indica que se mantiene la correlación entre las dos variables estudiadas, y la segunda, porque el valor numérico obtenido es mayor al obtenido en la primera prueba $(r=0,26)$, lo que interpretamos como el aprendizaje genuino del concepto de valencia química pese a que lo ideal hubiera sido que los coeficientes de correlación estuvieran más cerca de +/- 1 .

El valor obtenido al aplicar la correlación de Person a las variables tiempo de respuesta Vs número de aciertos, en la aplicación de las prueba con y sin ficha de características del concepto, aunque se considera una relación débil entre las variables analizadas, al menos indica la existencia de alguna relación y eso se interpreta como favorable para nuestra didáctica. Esta situación puede tener su origen en que la internalización profunda de los conceptos requiere de un mayor tiempo al que utilizamos en la presente investigación para su maduración; pero hay que reconocer que la teoría de la formación por etapas de las acciones mentales se hace evidente y funciona, al encontrar un aumento en el valor del coeficiente de correlación de Pearson, aplicado a los resultados de la prueba con ficha de características Vs prueba con Ficha sin características.

\section{CONCLUSIONES}

Del estudio los autores podemos concluir que: 1) La principal característica de la metodología utilizada fue considerar el tiempo como un indicador de internalización del concepto de valencia. 2) la técnica propuesta es fácil de utilizar, pues basta con relacionar la cantidad de aciertos que realiza cada estudiante en un tiempo determinado y así estratificar los resultados obtenidos en la prueba, tanto en el lenguaje para sí, como en la etapa mental propiamente dicha. 3) El éxito de la implementación de la TFEAM radica en que el docente sea asertivo al escoger la forma materializada del concepto, así como al definir la base orientadora de la acción.4) La metodología empleada para la enseñanza aprendizaje del concepto de valencia puede ser aplicada a otros conceptos de diferentes áreas del conocimiento.

\section{REFERENCIAS}

Angelo, T.A. y K.P. Cross, Classroom assessment techniques: A handbook for college teachers. Ann Arbor: National Center for the Improvement of Postsecondary Teaching and Learning, 2a Ed. In Classroom assessment techniques: A handbook for college teachers. San Francisco, USA (1993)

Borda, M., R. Tuesca y E. Navarro, Métodos cuantitativos: Herramienta para la investigación en salud. ECOE Ediciones, 228-234 (2005)

Galperín, P.Y., Teoría de la formación por etapas de las acciones mentales. Editorial MGY Moscú, Rusia (1995)

Gnitetskaya, T.N., Psychological basics for Arrangement of Stepwise Physics Module, Procedia - Social and Behavioral Sciences, 112(7), 382-386 (2014)

Rodríguez-Llerena, D. y J. Llovera-González, Estrategias de enseñanza en el laboratorio docente de Física para estudiantes de ingeniería, Lat. Am. J. Phys. Educ., 8(4), 1-8 (2014)

Rodríguez-Llerena, D. y J. Llovera-González, Estudio comparativo de las potencialidades didácticas de las simulaciones virtuales y de los experimentos reales en la enseñanza de la Física General para estudiantes universitarios de ciencias técnicas, Lat. Am. J. Phys. Educ., 4(1), 181-187 (2010)

Talízina, N., Psicología de la enseñanza, p57. Editorial Progreso, Moscú, Rusia (1988)

Tejada C, Gattas C y A. Villabona, Concepciones alternativas y errores conceptuales concepciones alternativas y errores conceptuales de estudiantes sobre modelos atómicos en química. Revista Entornos, 26(2), 267-274 (2013a)

Tejada, C., C. Chicangana y A. Villabona, Enseñanza de la química basada en la formación por etapas de acciones mentales (caso enseñanza del concepto de valencia), Revista Virtual Universidad Católica del Norte, 38, 143-157 (2013b)

Villarini, A., Manual para la enseñanza de destrezas de pensamiento. Proyecto de Educación Liberal Liberadora (PELL). San Juan, Puerto Rico (1991) 